\title{
ÍNDICES HEMATIMÉTRICOS E BIOQUÍMICA SANGUÍNEA NO CAVALO DE CAVALGADA EM CONDIÇÕES TROPICAIS
}

\author{
Stephânia Katurchi Mendes Melo ${ }^{1}$, Liliane Batista de LiRA ${ }^{2}$, TElga LuCEnA Alves Craveiro

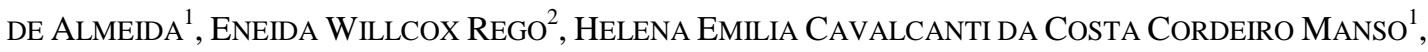 \\ HÉLIO CORDEIRO MANSO FILHO ${ }^{1}$ \\ ${ }^{1}$ Núcleo de Pesquisa Equina, Departamento de Zootecnia. Universidade Federal Rural de Pernambuco, Recife, \\ PE, Brasil. \\ ${ }^{2}$ Laboratório de Patologia Clinica, Departamento de Medicina Veterinária, Universidade Federal Rural de \\ Pernambuco, Recife,PE, Brasil \\ hmanso@dz.ufrpe.br
}

\section{RESUMO}

Objetivou-se por meio deste ensaio determinar as variáveis hematológicas (contagem de glóbulos vermelho, hemoglobina, hematócrito, volume corpúscular médio, hemoglobina corpuscular média, concentração hemoglobínica corpuscular média e coeficiente de variação da curva de distribuição das hemácias) e a bioquímica (proteínas plasmáticas totais, fibrinogênio, ureia, creatinina, ácido úrico, colesterol total $\mathrm{e}$ triglicérides) no sangue de cavalos de cavalgada. Para tanto, 46 equinos da raça Mangalarga Marchador, sendo 32 machos e 14 fêmeas, de idades diferentes $(6,83 \pm 0,65$ anos) foram avaliados. Os animais eram mantidos estabulados em diferentes centros de preparo de cavalos de cavalgada que apresentavam programa alimentar e regime de condicionamento similares. Avaliaram-se o grupo sexual (machos e fêmeas) e os grupos etários (menores de cinco anos, entre cinco e dez anos, maiores de dez anos). Os resultados indicaram diferenças na concentração hemoglobínica corpuscular média referente ao grupo sexual $(\mathrm{P}<0,05)$ e diferença na variação da concentração das proteínas plasmáticas totais referente aos grupos etários $(\mathrm{P}<0,05)$. Os resultados permitem concluir que não há diferenças importantes nos parâmetros bioquímicos e hematológicos em cavalo de cavalgada em relação ao sexo e à faixa etária, com exceção da concentração hemoglobínica corpuscular média e da concentração das proteínas plasmáticas totais, as quais, entretanto, não comprometem a performance física dos animais.

PALAVRAS-CHAVE: bioquímica; equino; eritrograma; esporte.

\section{HEMATOLOGICAL PARAMETERS AND BLOOD BIOCHEMISTRY IN PLEASURE HORSES IN TROPICAL REGION}

\section{ABSTRACT}

The objective of this study was to determine haematological variables (red blood cell count, hemoglobin, hematocrit, mean corpuscular volume, mean corpuscular hemoglobin, mean corpuscular hemoglobin concentration and coefficient of variation of the distribution curve of red blood cells) and blood biochemistry (total plasma proteins, fibrinogen, urea, creatinine, uric acid, total cholesterol and triglycerides) of pleasure horses. Therefore, we evaluated 46 Mangalarga Marchador horses, 32 males and 14 females at different ages $(6.83 \pm 0.65$ years). The animals were kept stabled at different pleasure horses training centers, which presented similar nutritional management and conditioning regimen. We evaluated the sex group (male and female) and age groups (younger than five years, between five and ten years, over ten years). The results indicated differences in 
the mean corpuscular hemoglobin concentration related to sexual group $(\mathrm{P}<0,05)$, and in the variation of total serum protein concentration $(\mathrm{P}<0,05)$ related to the age groups. The results suggested that there are important differences in biochemical and hematological parameters in horse

KEYWORDS: biochemistry; equine; erythrogram; sport.

\section{INTRODUÇÃO}

A raça Mangalarga Marchador é uma das mais importantes e a mais numerosa do país. Originária do sul de Minas Gerais (COSTA et al., 2005), surgiu do cruzamento de um garanhão Alter Real, presente de D. João VI ao Barão de Alfenas, com o rebanho mineiro da família Junqueira, de origem ibérica. A raça é prontamente identificada pelo seu andamento característico, a marcha, em substituição ao trote. Esse andamento está presente em poucas raças ao redor do mundo, mas é bastante apreciado no Brasil por cavaleiros que utilizam os seus animais para as cavalgadas.

O hemograma e os exames bioquímicos são exames complementares e constituem importantes ferramentas para o auxílio no diagnóstico de enfermidades na clínica médica equina (KANEKO, 1997), além de também serem úteis para avaliar aspectos da capacidade atlética dos equinos. O conhecimento dos constituintes sanguíneos constitui a base para a avaliação das alterações patológicas facilitando o diagnóstico de diferentes tipos de problemas nos animais (HARVEY et al., 1984). É sabido que os resultados dos exames laboratoriais podem sofrer alterações devido à idade, sexo, atividade física do animal, localização, entre outros fatores (MEYER \& HARVEY, 1998; ZHANG et al., 1998).

Diante do exposto, o objetivo deste ensaio foi determinar as concentrações das variáveis hematológicas e bioquímicas de cavalos condicionados para cavalgada em condições tropicais relacionando-as ao sexo e idade.

\section{MATERIAL E MÉTODOS}

O presente trabalho foi aprovado pelo Comitê de Ética da Universidade Federal Rural de Pernambuco, sob o número 23082.007851/2007.

Foram utilizados 46 equinos da raça Mangalarga Marchador, sendo 32 machos e 14 fêmeas, de idades diferentes $(6,83 \pm 0,65$ anos $)$ e estabulados em três diferentes centros de preparação de cavalos de cavalgada na região metropolitana do Recife - PE ( $8^{\circ} 3^{\prime} 0^{\prime \prime} \mathrm{S}$; 34 $\left.33^{\circ} 44^{\prime \prime} \mathrm{O}\right)$. Os animais recebiam programa alimentar similar, constituindo- riding toward sex and age group, except for mean corpuscular hemoglobin concentration and total plasma proteins concentration, which do not compromise physical performance of these animals.

se do fornecimento de concentrado comercial (aproximadamente 5,0kg/dia/animal; $14,0 \%$ de protína bruta, 3,5\% de extrato etéreo e 2,9 Mcal de energia digestível), capim elefante (Pennisetum purpureum Schumach) in natura e picado em forrageira (aproximadamente 20,0kg/dia/animal), sal mineralizado (Coequi Plus - Tortuga $\left.{ }^{\circledR}\right)$ e água $a d$ libitum. Os animais apresentavam regime de condicionamento físico similar, o qual consistia de pelo menos três cavalgadas semanais ao passo e à marcha (picada ou batida), com distâncias de 10 a $15 \mathrm{~km}$, em pisos variados e em terrenos com altitude variando de 50 até 150 metros acima do nível do mar. A carga transportada (massa do cavaleiro mais a dos arreios de montada e condução) não ultrapassava $100 \mathrm{~kg}$.

Para análise, os animais foram agrupados em grupo sexual (masculino, castrados e intactos, e feminino) e grupo etário (grupo I: animais com menos de cinco anos; grupo II: animais entre cinco e dez anos; e grupo III: animais com mais de dez anos).

Para a determinação do escore corporal dos animais utilizou-se o método descrito por COSTA et al. (2001), que possui escala de 1 até 7 , sendo 1 o animal caquético e 7 o animal obeso.

Amostras de sangue foram colhidas por venóclise na julgular, em tubos a vácuo (BD Vacuntainer $\left.{ }^{\circledR}\right)$ com anticoagulante, com os animais em repouso, sendo acondicionadas sob refrigeração e enviadas ao laboratório para a realização das análises. O sangue total foi utilizado para a realização das análises hematológicas: contagem de glóbulos vermelhos (CGV), hemoglobina (HB), hematócrito (HT), volume corpuscular médio (VCM), concentração hemoglobínica corpuscular média (CHCM) e amplitude de distribuição dos eritrócitos coeficiente de variação (RDW-CV), sendo determinadas em analisador hematológico automatizado (Analisador Hematológico Veterinário pocH-100iV Diff, Sysmex®). Posteriormente, as amostras foram centrifugadas (3000 rotações por minuto durante cinco minutos) para a obtenção do plasma e realização das análises bioquímicas: ureia (UREIA), creatinina (CREAT), ácido úrico (AcURI), colesterol total (COLE-T) e triglicérides (TRIG), sendo determinadas por meio do uso de kits 
comerciais (DOLES $®$ Reagente) em equipamento de bioquímica semi-automático (Doles D250, DOLES $\left.{ }^{\circledR}\right)$. A determinação da proteína plasmática total (PPT) foi realizada por meio da refratometria manual e a determinação do fibrinogênio (FIBRI) foi realizada por meio da técnica de precipitação pelo calor em banho-maria a $57^{\circ} \mathrm{C}$ durante três minutos (KANEKO et al., 1998).

Para a análise estatística, todos os resultados foram submetidos ao teste $\mathrm{T}$ ou ANOVA com $\mathrm{P}$ estabelecido em $5 \%$ e, quando necessário, ao teste Holm-Sidak como post doc com $\mathrm{P}$ também estabelecido em 5\% (SigmaStat ${ }^{\circledR}$ 3.0).

\section{RESULTADOS E DISCUSSÃO}

Verificou-se diferença nos valores de
CHCM, quando os resultados foram avaliados conforme o grupo sexual, sendo nos machos mais baixo que nas fêmeas (Tabela 1), mas não foram observadas diferenças nos demais parâmetros avaliados (Tabela 2). Quando os resultados foram avaliados conforme os grupos etários, foram observadas diferenças $(\mathrm{P}<0,05)$ na concentração da PPT, com valores mais baixos nos animais do grupo I (com menos de cinco anos) do nos animais dos demais grupos (Tabela 3), mas não foram observadas diferenças nas demais variáveis (Tabela 4) para o mesmo grupo. As tabelas 1 e 2 apresentam os resultados obtidos nas variáveis analisadas conforme o grupo sexual e as Tabelas 3 e 4, os resultados obtidos nas variáveis analisadas conforme os grupos etários.

Tabela 1. Média e desvio padrão do índice do escore corporal, hematológicos e hematimétricos conforme o grupo sexual de equinos da raça Mangalarga Marchador na região metropolitana do Recife-PE

\begin{tabular}{lcc}
\hline \multirow{2}{*}{ Variáveis Analisadas } & \multicolumn{2}{c}{ Grupo sexual } \\
\cline { 2 - 3 } & MACHO $(\mathrm{N}=32)$ & FÊMEA $(\mathrm{N}=14)$ \\
\hline Escore Corporal & $4,00 \pm 0,10$ & $4,18 \pm 0,13$ \\
CGV $(\mathrm{X} \mathrm{10} / \mathrm{L})$ & $8,20 \pm 0,23$ & $7,95 \pm 0,43$ \\
Hemoglobina (g/dL) & $12,62 \pm 0,29$ & $12,86 \pm 0,58$ \\
Hematócrito (\%) & $36,0 \pm 0,01$ & $34,0 \pm 0,14$ \\
VCM (Fl) & $43,88 \pm 0,40$ & $43,8 \pm 0,94$ \\
CHCM $(\mathrm{g} / \mathrm{dl})$ & $35,43 \pm 0,13^{\mathrm{A}}$ & $36,13 \pm 0,31^{\mathrm{B}}$ \\
RDW-CV $(\%)$ & $19,89 \pm 0,18$ & $19,67 \pm 0,32$ \\
\hline
\end{tabular}

Observações: CGV: contagem de glóbulos vermelhos; VCM: volume corpuscular médio; CHCM: concentração de hemoglobina corpuscular média; RDW: coeficiente de variação do tamanho médio das hemácias. Letras diferentes na mesma linha indicam que $\mathrm{P}<0,05$ pelo teste $\mathrm{T}$.

Tabela 2. Média e desvio padrão da concentração das proteínas plasmáticas totais, fibrinogênio, ureia, creatinina, ácido úrico, colesterol total e triglicérides conforme o grupo sexual de equinos da raça Mangalarga Marchador da região metropolitana do Recife-PE

\begin{tabular}{lcc}
\hline Variáveis Analisadas & Grupo sexual & \\
\cline { 2 - 3 } & MACHO $(\mathrm{N}=32)$ & FÊMEA $(\mathrm{N}=14)$ \\
\hline Proteínas plasmáticas totais $(\mathrm{g} / \mathrm{L})$ & $69,87 \pm 8,30$ & $67,54 \pm 14,50$ \\
Fibrinogênio $(\mathrm{g} / \mathrm{L})$ & $3,74 \pm 0,42$ & $4,00 \pm 0,88$ \\
Ureia $(\mathrm{mmol} / \mathrm{L})$ & $4,06 \pm 0,48$ & $3,49 \pm 0,21$ \\
Creatinina $(\mu \mathrm{mol} / \mathrm{L})$ & $92,76 \pm 2,27$ & $96,48 \pm 5,87$ \\
Ácido úrico $(\mathrm{mmol} / \mathrm{L})$ & $0,031 \pm 0,002$ & $0,033 \pm 0,002$ \\
Colesterol total $(\mathrm{mmol} / \mathrm{L})$ & $2,28 \pm 0,15$ & $2,67 \pm 0,38$ \\
Triglicérides $(\mathrm{mmol} / \mathrm{L})$ & $0,31 \pm 0,02$ & $0,32 \pm 0,05$ \\
\hline
\end{tabular}

Observação: letras diferentes na mesma linha indicam que $\mathrm{P}<0,05$ pelo teste $\mathrm{T}$.

Os valores encontrados no presente estudo assemelham-se aos valores descritos na literatura para animais sadios em diferentes condições (KANEKO et al., 1998; PERRY, 2009), mesmo estando os animais estudados em região tropical e sendo manejados de modo particular. Deve-se ressaltar que esses resultados representam um grupo específico de cavalos de trabalho que está amplamente difundido no país. Esses animais, todos da raça Mangalarga Marchador, não são utilizados 
em provas regulares de marcha e sim em cavalgadas, que exigem dos animais um condicionamento para exercícios de baixa a média intensidade e longa duração.

Tabela 3. Média e desvio padrão da concentração das proteínas plasmáticas totais, fibrinogênio, ureia, creatinina, ácido úrico, colesterol total e triglicérides conforme o grupo etário de equinos da raça Mangalarga Marchador da região metropolitana do Recife-PE

\begin{tabular}{lccc}
\hline & \multicolumn{3}{c}{ Grupo Etário } \\
\cline { 2 - 4 } Variáveis Analisadas & GRUPO I & GRUPO & GRUPO III \\
& $(\mathrm{N}=18)$ & II $(\mathrm{N}=18)$ & $(\mathrm{N}=10)$ \\
\hline Proteínas plasmáticas totais $(\mathrm{g} / \mathrm{L})$ & $66,82 \pm 1,06^{\mathrm{B}}$ & $70,25 \pm 1,34^{\mathrm{A}}$ & $71,40 \pm 0,94^{\mathrm{A}}$ \\
Fibrinogênio $(\mathrm{g} / \mathrm{L})$ & $4,00 \pm 0,55$ & $3,82 \pm 0,68$ & $3,33 \pm 0,99$ \\
Ureia $(\mathrm{mmol} / \mathrm{L})$ & $3,80 \pm 0,62$ & $4,03 \pm 0,61$ & $3,81 \pm 0,25$ \\
Creatinina $(\mu \mathrm{mol} / \mathrm{L})$ & $88,55 \pm 3,55$ & $94,41 \pm 3,07$ & $102,54 \pm 6,24$ \\
Ácido úrico $(\mathrm{mmol} / \mathrm{L})$ & $0,034 \pm 0,0031$ & $0,030 \pm 0,0016$ & $0,033 \pm 0,0031$ \\
Colesterol total $(\mathrm{mmol} / \mathrm{L})$ & $2,34 \pm 0,18$ & $2,48 \pm 0,31$ & $2,38 \pm 0,33$ \\
Triglicérides $(\mathrm{mmol} / \mathrm{L})$ & $0,36 \pm 0,03$ & $0,27 \pm 0,03$ & $0,31 \pm 0,05$ \\
\hline
\end{tabular}

Observações: Grupos etários: grupo I - menos de cinco anos; grupo II - entre cinco e dez anos; grupo III - mais de dez anos. Letras diferentes na mesma linha indicam que $\mathrm{P}<0,05$ pelo teste de Holm-Sidak.

Tabela 4. Média e desvio padrão do índice do escore corporal, hematológico e hematimétricos conforme o grupo etário de equinos da raça Mangalarga Marchador na região metropolitana do Recife-PE

\begin{tabular}{lccc}
\hline \multirow{2}{*}{ Variáveis Analisadas } & \multicolumn{3}{c}{ Faixa etária } \\
\cline { 2 - 4 } & $\begin{array}{c}\text { GRUPO I } \\
(\mathrm{N}=18)\end{array}$ & $\begin{array}{c}\text { GRUPO II } \\
(\mathrm{N}=18)\end{array}$ & $\begin{array}{c}\text { GRUPO III } \\
(\mathrm{N}=10)\end{array}$ \\
\hline Escore Corporal & $4,03 \pm 0,15$ & $4,03 \pm 0,06$ & $4,15 \pm 0,20$ \\
CGV $\left(\mathrm{X} 10^{3} / \mathrm{L}\right)$ & $8,58 \pm 0,38$ & $7,95 \pm 0,30$ & $7,58 \pm 0,25$ \\
Hemoglobina (g/dL) & $12,94 \pm 0,47$ & $12,33 \pm 0,37$ & $12,78 \pm 0,51$ \\
Hematócrito $(\%)$ & $36,0 \pm 0,01$ & $35,0 \pm 0,01$ & $34,0 \pm 0,01$ \\
VCM (Fl) & $42,82 \pm 0,58$ & $44,27 \pm 0,62$ & $44,27 \pm 0,62$ \\
CHCM (g/dL) & $35,48 \pm 0,22$ & $35,51 \pm 0,22$ & $36,10 \pm 0,28$ \\
RDW-CV $(\%)$ & $20,08 \pm 0,27$ & $19,92 \pm 0,23$ & $19,16 \pm 0,30$ \\
\hline
\end{tabular}

Observações: Grupos etários: grupo I - menos de cinco anos; grupo II - entre cinco e dez anos; grupo III - mais de dez anos. CGV: contagem de glóbulos vermelhos; VCM: volume corpuscular médio; CHCM: concentração de hemoglobina corpuscular média; RDW-CV: coeficiente de variação do tamanho médio das hemácias. Letras diferentes na mesma linha indicam que $\mathrm{P}<0,05$ pelo teste de Holm-Sidak.

As variações observadas neste experimento tanto nas comparações entre os grupos sexuais como entre os grupos etários entre os parâmetros analisados não eram esperadas, corroborando com o estudo realizado por NEVES et al. (2005), pois todos os animais avaliados eram animais adultos e possuiam programa alimentar e regime de condicionamento similares. É importante ressaltar que os valores médios observados para as variáveis analisadas encontravam-se no intervalo de normalidade para equinos (KANEKO et al., 1998; PERRY, 2009). Tais achados confirmam o bom estado de saúde dos animais avaliados e poderão servir como referência para equinos de sela em condições semelhantes.

As avaliações feitas neste experimento revelaram diferenças no $\mathrm{CHCM}(\mathrm{P}<0,05)$, sendo o maior valor observado nas fêmeas, discordando de VEIGA et al. (2006), que analisaram os valores hematológicos de 142 equinos da raça Crioula e observaram CHCM maior nos machos. Todavia, os resultados concordam com VEIGA et al. (2006), que verificaram maiores valores de $\mathrm{CHCM}$ para animais de corrida, o que é explicado pela necessidade de maior aporte de oxigênio aos tecidos em elevado metabolismo, e divergem dos achados de CONCEIÇÃO et al. (2001), que estudou equinos da raça Quarto de Milha.

Os valores do CHCM associam-se à maior ou menor percentagem do hematócrito, cujas variações, de acordo com diferentes autores, relacionam-se com a realização de atividade física, como resultado da mobilização de reservas do sangue do baço e também da desidratação que se 
desenvolve nos exercícios de longa duração, como as cavalgadas (TEIXEIRA NETO, 2004; PICCIONE et al., 2007; PUOLI FILHO et al., 2007; BELLI, 2008).

Valores mais elevados para hemoglobina e hematócrito foram apresentados pelos cavalos do Grupo I, com menos de cinco anos, quando comparados aos demais grupos etários, mas sem diferenças estatísticas, apesar de os animais deste experimento serem frequentemente manejados e estarem em ambiente calmo. De acordo com DUNCAN et al. (1994), animais muito jovens, por serem mais excitáveis, podem apresentar alguns valores hematológicos mais elevados. Por outro lado, vários estudos (HARVEY et al., 1984; DINEV \& KHUBENOV, 1986; ALMEIDA \& SILVA, 1995; MEYER \& HARVEY, 1998; VEIGA et al., 2006) detectaram redução na percentagem do hematócrito e na concentração da hemoglobina de animais jovens quando comparados com animais adultos. Credita-se essa variação à maior hemodiluição nos animais jovens. No atual estudo, o volume plasmático não foi avaliado, o que poderia ser importante para se determinar o real valor dos índices hematimétricos pesquisados nos cavalo de cavalgada.

Não foram verificadas diferenças significativas nos valores do hematócrito quanto ao sexo, concordando com resultados descritos por DINEV \& KHUBENOV (1986), mas opondo-se aos achados de VAN HEERDEN et al. (1990), que observaram maior valor de hematócrito nas fêmeas. CEBULJ-KADUNC et al. (2002) também detectaram maiores valores de hemoglobina para machos, o que não foi verificado neste trabalho, corroborando VEIGA et al. (2006).

Observa-se que os valores do RDW, que avalia o grau de heterogenicidade dos eritrócitos, foi semelhante ao descrito por EASLEY et al. (1985), porém inferior aos descritos para cavalos em treinamento e para potros da raça Puro Sangue Inglês (BALARIN et al., 2006), tanto quando comparados com os animais agrupados pelo sexo como pela faixa etária. No presente estudo não foram observadas diferenças entre os grupos sexuais, corroborando o estudo de BALARIN et al. (2001).

Foram detectadas diferenças na concentração das proteínas plasmáticas totais quando os animais foram comparados conforme o grupo etário, sendo os valores mais elevados encontrados nos cavalos com mais de 10 anos (aproximadamente 71,5 $\mathrm{g} / \mathrm{L}$ ). Os resultados relacionam-se com a senilidade, pois é largamente conhecido que animais mais jovens apresentam maior quantidade de água corporal e que, com a senilidade, esse acúmulo vai reduzindo e com isso pode haver modificação na concentração das proteínas plasmáticas totais (MANSO FILHO et al., 2008). Também é importante recordar que estudos envolvendo exercícios de longa distância e diferentes graus de intensidade revelaram aumentos de proteínas do plasma após exercícios (SANTOS et al., 2001; SANTOS, 2006; PUOLI FILHO et al., 2007). Analisando-se a concentração das proteínas plasmáticas totais presentes no sangue, não foram observadas diferenças na concentração do fibrinogênio, indicando um bom estado de saúde dos animais, já que variações na concentração plasmática do fibrinogênio podem contribuir de forma positiva e precoce para o diagnóstico de estados inflamatórios e infecciosos (DI FILLIPO et al., 2009).

Avaliações das concentrações de ureia e creatinina podem ser utilizadas para avaliar o metabolismo proteico e também a função renal dos animais. Apesar da sua importância, poucos trabalhos na literatura avaliam a influência dos fatores sexuais e idade sobre tais parâmetros. Foi observado que as concentrações da ureia, creatinina e ácido úrico encontravam-se no intervalo de valores de normalidade para a espécie, e se assemelham aos resultados descritos na literatura (KANEKO et al., 1998).

Estudo realizado por WANDERLEY et al. (2010) com animais da mesma raça revelou concentrações médias de ureia $(4,0 \mathrm{mmol} / \mathrm{L})$ e creatinina (94 mmol/L) nos animais em jejum, similares às descritas neste estudo. ROSE et al. (1979), BAUER et al. (1984) e EDWARDS et al. (1989), apesar de terem utilizado delineamentos diferentes para avaliar a influência do fator etário sobre as taxas de creatinina, observaram a influência da evolução da idade sobre a variabilidade dos teores desse componente. Finalmente deve-se recordar que o tipo de manejo e alimentação pode influenciar nos valores desses marcadores do metabolismo proteico nos animais. Além disso, deve-se ressaltar que os resultados deste estudo assemelham-se aos de NEVES et al. (2005), que estudaram equinos da raça Mangalarga Paulista, clinicamente sadios e separados conforme faixa etária e sexo. Os valores encontrados em ureia e creatinina conforme o grupo sexual e o grupo etário foram semelhantes.

As reservas de lipídios no sangue dos cavalos podem ser avaliadas pela determinação da concentração de triglicérides e colesterol total; todavia, o triglicérides é o mais importante deles, pois é fonte de energia para os animais atletas e em outras condições. Os valores encontrados para a concentração do colesterol total e triglicérides 
assemelham aos descritos tanto como valores de referência, independente da raça (KANEKO et al., 1998; PERRY, 2009), como para cavalos da raça Mangalarga Marchador (WANDERLEY et al., 2010). Os cavalos de cavalgada não recebiam nenhum tipo de suplemento ou de concentrado com elevada percentagem de gordura, mas estavam com índice de escore corporal em torno de 4 , indicando um boa reserva corporal de gordura.

Os lipídeos presentes no sangue são importantes para os animais que realizam exercícios de baixa intensidade e com duração de baixa a média, que levam a uma significante utilização das gorduras como fonte de energia para o trabalho muscular.

Os resultados obtidos para a concentração do triglicérides são semelhantes aos de equinos em repouso pós-exercícios, conforme reportado por WANDERLEY et al. (2010); todavia, os valores da concentração do colesterol total apresentam-se maiores, discordando do estudo descrito por SLOET VAN OLDRUITENBORGH-OOSERBAN et al. (2002), que observaram que a concentração do colesterol total reduziu significativamente após o exercício em cavalos alimentados com dietas contendo $1,5 \%$ de extrato etéreo.

Em estudo realizado por HYYPPÄ (2005), a recuperação das concentrações séricas do triglicérides foi lenta pós-exercício. Segundo o mesmo autor, uma hora após o término do exercício ocorre aumento na liberação de insulina, que, por sua vez, inibe a lipólise num momento em que as concentrações séricas de lipídeos já estariam baixas em função de seu uso como fonte energética. Esse aumento da concentração do triglicérides é esperado em consequência do bloqueio na ação da insulina e do efeito hiperglicemiante gerado pelas catecolaminas e cortisol circulantes frente ao esforço físico. Com isso, ocorre um balanço energético negativo, semelhante ao que ocorre quando um equino é submetido a jejum alimentar, havendo lipólise e mobilização de outras fontes energéticas (DURHAM, 2006; DUGAT et al., 2010).

\section{CONCLUSÕES}

Equinos da raça Mangalarga Marchador utilizados em provas de cavalgada não apresentam alterações hematológicas e/ou bioqímicas, quando avaliados segundo sexo e idade. As diferenças observadas para a variavel CHCM, com relacao ao grupo sexual e PPT para grupo etário não comprometem o desempenho físico dos animais.

\section{AGRADECIMENTOS}

Aos criadores e proprietários do cavalo Mangalarga Marchador de Pernambuco, à GUABI Nutrição Animal, Goiana-PE, e à AJINOMOTO do Brasil, São Paulo-SP, pelo apoio financeiro a este projeto.

\section{REFERÊNCIAS}

ALMEIDA, M. A. Z.; SILVA, N. M. Determinação dos valores hematológicos normais do cavalo (Equus caballus, Linnaeus) da raça Crioula. A Hora Veterinária, v.15, n.87, p.48-50, 1995.

Balarin, M. R. S.; Fonteque, J. H.; Souza, C.; Saito. M. E.; Kohayagawa, A.; Lopes, R. S. Valores da amplitude de distribuição do tamanho dos eritrócitos (RDW - Red Cell Distribution Width) em eqüinos da raça puro sangue inglês (PSI) de ambos os sexos de 12 a 24 meses de idade. Semina: Ciências Agrárias, v.22, n.2, p.135-137, 2001.

BALARIN, M. R. S.; LOPES, R. S.; KOHAYAGAWA, A.; LAPOSY, C. B.; FONTEQUE, J. H. Valores da Amplitude de Distribuição do Tamanho dos Eritrócitos (RDW) em eqüinos Puro Sangue Inglês (PSI) submetidos a exercícios de diferentes intensidades. Brazilian Journal of Veterinary Research and Animal Science, v.43, n.5, p.637-641, 2006.

BAUER, J. E.; HARVEY, J. W.; ASQUITH, R. L.; McNULTY, P. K.; KIVIPELTO, J. Clinical chemistry reference values of foals during the first years of life. Equine Veterinary Journal, v.16, n.4, p.361-363, 1984.

BELLI, C. B.; MICHIMA, L. E. S.; LATORRE, S. M.; FERNANDES, W. R. Solução concentrada de albumina equina na fluidoterapia em equinos com desidratação leve a moderada. Arquivos Brasileiros de Medicina Veterinária e Zootecnia, v.60, n.1, p.30-35, 2008.

CEBULJ-KADUNC, N.; BOZIC, M.; KOSEC, M.; CESTNIK, V. The influence of age and gender on hematological parameters in Lippizan horses. Journal of veterinary medicine. A, Phisiology, pathology, clinical medicine, v.49, n.4, p.217-221, 2002.

CONCEIÇÃO, M.; LAPOSY, C. B.; MELCHERT, A.; LOPES, R. S.; KOHAYAGAWA, A.; TAKAHIRA, R. K. Hemograma e bioquímica sérica de eqüinos da raça Quarto de Milha antes e após o exercício. Veterinária Notícias, v.7, n.2, p.87-92, 2001.

COSTA, M. D.; BERGMANN, J. A. G.; RESENDE, A. S. C.; FONSECA, C. G.; FARIA, F. J. C. Estudo da subdivisão genética da raça Mangalarga Marchador. Arquivo Brasileiro de Medicina Veterinária e Zootecnia, v.57, n.2, p.272-280, 2005.

DI FILLIPO, P. A.; SANTANA, A. E.; REZENDE, L. G. Estudo comparativo entre as técnicas de Schalm e de Clauss na determinação da concentração plasmática do fibrinogênio em equinos hígidos e com cólica. Ciência 
Animal Brasileira, v.10, n.4, p.1231-1236, 2009.

DINEV, D.; KHUBRNOV, KhD. Normal values of the hematological, biochemical and enzymological indices of the donkey. Veterinarno Meditsinski Nauki, v.23, n.10, p.69-75, 1986

DUNCAN, J. R.; PRASSE, K. W.; WAHAFFEY, E. A. Veterinary laboratory medicine: Clinical Pathology. 3ed. Iowa: Iowa State University, 1994. 300p.

DUGAT, S. L.; TAYLOR, T. S.; MATTHEWS, N. S.; GOLD, J. R. Values for triglycerides, insulin, cortisol and ACTH in a herd of normal donkeys. Journal of Equine Veterinary Science, v.30, n.3, p.141-144, 2010.

DURHAM, A. E. Clinical application of parenteral nutrition in the treatment of five ponies and one donkey with hyperlipaemia. Veterinary Record, v.158, n.5, p.159-164, 2006.

EASLEY, J. R. Erytrhogram and red cell distribution width of equidae with experimentally induced anemia. American Journal of Veterinary Research, v. 46, n.11, p.2378-84, 1985.

EDWARDS, D. J.; BROWNLOW, M. A.; HUTCHINS, $P$. R. Indices of renal function: reference values in normal horses. Australian Veterinary Journal, v.66, n.2, p.6063, 1989

HARVEY, J. W.; ASQUITH, R. L.; McNULTHY, P. K.; KIVIPELTO, J.; BAUER, J. E. Haematology of foals up to one year old. Equine Veterinary Journal, v.16, n.4, p.347-353. 1984.

HYYPPÄ, S. Endocrinal responses in exercising horses. Livestock Production Science, v.92, p.113-121, 2005.

KANEKO, J. J.; HARVEY, J. W.; BRUSS, M. L. Clinical biochemistry of domestic animals. San Diego: Academic Press, 1997. 932 p.

KANEKO, J. J.; HARVEY, J. W.; BRUSS, M. L. Clinical Biochemistry of Domestic Animals. San Diego: Academic Express, 6. ed., 1998. 936 p.

MANSO FILHO, H. C.; ABREU, J. M.; MANSO, H. E. C. C. C. Suplementação com aminoácidos e nutracêuticos modifica a composição corporal e parâmetros sangüíneos de eqüinos idosos. Revista do Conselho Federal de Medicina Veterinária, v.43, p.9-15, 2008.

MEYER, D. J.; HARVEY, J. W. Veterinary laboratory medicine: interpretation and diagnosis. 2. ed Philadelphia: W.B. Saunders Company, 1998. 373 p.

NEVES, M.; BENESI, F. J.; NORONHA, T.; COELHO, C. S.; SOUZA, P. M.; MIRANDOLA, R. M. S.; FERNANDES, W. R. Função Renal em equinos sadios da raça Mangalarga Paulista, criados no estado de São Paulo. Revista Brasileira de Ciência Veterinária, v.12, n.1/3, p.106-109, 2005.

PERRY, B. W. Clinical Pathology references data. IN: ROBINSON, N. E.; SPRAUBERRY, K. A. Current Therapy in Equine Medicine. Filadelfia: Saunders, 6th ed., 2009, p.956-980.
PICCIONE, G.; GIANNETTO, C.; FAZIO, F.; DI MAURO, S.; CAOLA, G. Haematological response to different workload in jumper horses. Bulgarian Journal of Veterinary Medicine, Prague, v.10, n.4, p.21-28, 2007.

PUOLI FILHO, J. N. P.; NETO, T. L. B.; RODRIGUES, P. H. M.; GARCIA, H. P. L. Parâmetros fisiológicos do desempenho de cavalos de alta performace hidratados voluntariamente com água ou soluções isotônicas contendo carboidrato. Brazilian Journal Veterinary Research Animal Science, São Paulo, v.44, n.2, p.122$131,2007$.

REVINGTON, M. Haematology of the racing Thoroughbred in Australia 1: reference values and the effect of excitement. Equine Veterinary Journal, v.15, n.2, p.141-144, 1983.

ROSE, R. J.; BACKHOUSE, W.; CHAN, W. Plasma biochemistry changes in the thoroughbred foals during the first 4 weeks of life. Journal of Reproduction and Fertility, v.27, Suppl., p.601-605, 1979.

SANTOS, S. A.; SILVA, R. A. M. S.; AZEVEDO, J. R. M.; MELLO, M. A. R.; SOARES, A. C.; SIBUYA, C. Y.; ANAMURA, C. A. Alterações séricas de eletrólitos e alterações da proteína total em Cavalo Pantaneiro durante muito tempo de exercício distância. Arquivo Brasileiro de Medicina Veterinária e Zootecnia, v.53, n.3, p.351$357,2001$.

SANTOS, V. P. Efeito do protocolo de exercício sobre variáveis hematológicas e bioquímicas em equinos de salto. Acta Scientiae Veterinarie, v.34, n.2, p.243-244, 2006.

SLOET VAN OLDRRUITNBORGH-OOSTERBAN, M. M.; ANNEE, M. P.; VERDEGAAL, E. J.; LEMMENS, A. G.; BEYNEN, A. C. Exercise and metabolismassociated blood values variables in Standardbred fed either a low or a high fat diet. Equine Veterinary Journal, London, v.34, p.29-32, 2002.

TEIXEIRA NETO, A. R., FERRAZ, G. C.; MATAQUEIRO, M. I.; LACERDA-NETO, J. C.; QUEIROZ-NETO, A. Reposição eletrolítica sobre as variáveis fisiológicas de cavalos em prova de enduro de 30 e 60 km. Ciência Rural, v.34, n.5, p.1505-1511, 2004.

VAN HEERDEN, J.; DAUTH, J.; DREYER, M. J.; NICHAS, E.; MARSHALL, C.; DeWAAL, D. T. Selected laboratory parameters of thoroughbreds. Journal of South African Veterinary Association, v.61, n.4, p.155-158, 1990.

VEIGA, A. P. M.; LOPES, S. T. A.; FRANCISCATO, C.; OLIVEIRA, L. S. S.; MERINI, L. P. Valores hematológicos, PPT e fibrinogênio do cavalo crioulo Suas variações em relação ao sexo, idade e manejo. Acta Scientiae Veterinariae, v.34, p.275-279, 2006.

WANDERLEY, E. K.; MANSO FILHO, H. C.; MANSO, H. E. C. C.; SANTIAGO, T. A.; McKEEVER, K. H. Metabolic changes in four-beat gaited horses after field marcha simulation. Equine Veterinary Journal, v.42, 
n.38, p.105-109, 2010.

clinical chemistry laboratory results. Clinical Chemistry,

ZHANG, D. J.; ELSWICK, R. K.; MILLER, W. G.;

v.44, n.6, p.1325-1333, 1998.

BAILEY, J. L. Effect of serum-clot contact time on

Protocolado em: 06 dez. 2011. Aceito em: 28 fev. 2013 